\title{
Highly efficient in vitro translation of authentic affinity-purified messenger ribonucleoprotein complexes
}

\author{
SARAH E. FRITZ, NAZMUL HAQUE, and J. ROBERT HOGG \\ Biochemistry and Biophysics Center, National Heart, Lung, and Blood Institute, National Institutes of Health, Bethesda, Maryland 20892, USA
}

\begin{abstract}
Cell-free systems are widely used to study mechanisms and regulation of translation, but the use of in vitro transcribed (IVT) mRNAs as translation substrates limits their efficiency and utility. Here, we present an approach for in vitro translation of messenger ribonucleoprotein (mRNP) complexes affinity purified in association with tagged mRNAs expressed in mammalian cells. We show that in vitro translation of purified mRNPs is much more efficient than that achieved using standard IVT mRNA substrates and is compatible with physiological ionic conditions. The high efficiency of affinity-purified mRNP in vitro translation is attributable to both copurified protein components and proper mRNA processing and modification. Further, we use translation inhibitors to show that translation of purified mRNPs consists of separable phases of run-off elongation by copurified ribosomes and de novo initiation by ribosomes present in the translation extracts. We expect that this in vitro system will enhance mechanistic studies of eukaryotic translation and translation-associated processes by allowing the use of endogenous mRNPs as translation substrates under physiological buffer conditions.
\end{abstract}

Keywords: in vitro translation; RNA affinity purification; mRNP; Pseudomonas phage 7; RNA-binding protein; RNP purification

\section{INTRODUCTION}

Eukaryotic protein synthesis is a dynamic molecular process that occurs in three stages: initiation, elongation, and termination/recycling. Cell-free protein expression systems have long been used to study mechanisms and regulation of each step of mRNA translation. Traditional in vitro translation protocols involve incubation of protein-free in vitro transcribed (IVT) mRNAs in translation-competent cytoplasmic extracts (Katzen et al. 2005). The application of RNA interference and CRISPR/Cas technology now allows for relative ease in targeted manipulation of trans-acting factors within cell-free translation systems, bolstering their utility for understanding contributions of individual proteins (Rakotondrafara and Hentze 2011). However, existing protocols may fail to recapitulate important mechanistic and regulatory features of eukaryotic translation as they lack mRNA substrates that have undergone proper assembly into ribonucleoprotein complexes (mRNPs).

In all cells, dynamic RNA-protein associations are responsible for carrying out mRNA function and regulation (Moore 2005). RNA-binding proteins have been found to impact each stage of translation, from initiation to recycling (Gehring et al. 2017). Moreover, the ribosome engages in extensive communication with additional mRNP components to determine

Corresponding author: j.hogg@nih.gov 118.

Article is online at http://www.rnajournal.org/cgi/doi/10.1261/rna.065730. rates of mRNA decay (Shoemaker and Green 2012). As mRNP assembly is a context-dependent, highly orchestrated process (Mitchell and Parker 2014), it is important to study mRNPs that have undergone physiological biogenesis in order to accurately study mRNA translation and decay. In addition to the possibility that nonphysiologically assembled mRNPs may fail to recapitulate important processes, previous work has suggested that poor in vitro translation efficiency could in part be due to inappropriate assembly of transcripts into mRNPs upon addition of nonphysiological, IVT mRNA into highly concentrated translation extracts (Panthu et al. 2015). Components of mRNPs could enhance translation through direct mechanisms, such as the role of the exon junction complex protein MLN51 in stimulating translation by engaging eIF3 (Chazal et al. 2013), or by serving more general structural functions that are currently not well understood (Panthu et al. 2015). Thus, a cell-free protein expression system is needed in which endogenously assembled mRNP complexes can be isolated from cells and introduced as substrates for in vitro translation.

RNA-based affinity purification systems have been valuable tools for the isolation and characterization of endogenously assembled RNPs (Slobodin and Gerst 2010; Yoon and Gorospe 2016; Panchapakesan et al. 2017). In particular, a system based on the Pseudomonas phage 7 (PP7) coat protein and its cognate hairpin binding site has been used to

This is a work of the US Government. 
investigate proteins associated with noncoding RNAs and the targeted degradation of transcripts by the nonsense-mediated mRNA decay pathway (Hogg and Collins 2007a,b; Hogg and Goff 2010; Ge et al. 2016). The primary use of such techniques has involved identification of mRNP components by mass spectrometry, but the ability to isolate endogenous mRNPs under conditions optimized to maintain RNAprotein interactions raises the possibility that affinity-purified mRNPs may also be used for downstream functional assays.

Here we demonstrate that affinity-purified mRNP complexes can be substrates for efficient cell-free protein synthesis. By monitoring production of NanoLuciferase (NLuc) protein from PP7-purified mRNPs (Hall et al. 2012), we show that affinity-purified mRNPs are rapidly translated in cytoplasmic extracts prepared from human cell lines (Rakotondrafara and Hentze 2011). A comparison to standard IVT mRNA substrates reveals a greater than 100-fold translation preference for correctly packaged mRNPs. Notably, maximal mRNP translation activity occurs in physiological $\mathrm{K}^{+}$and $\mathrm{Mg}^{2+}$ ionic concentrations, in contrast to the nonphysiological salt requirements of IVT mRNAs. Further, use of standard small molecule inhibitors of translation, harringtonine and puromycin, indicates that the translation activity of purified mRNPs arises from both elongation by copurified ribosomes and de novo initiation in extracts. We expect our in vitro system will provide a versatile tool to study contributions of mRNA-binding proteins and RNA modifications to protein synthesis and translation-dependent processes under cellular ionic conditions.

\section{RESULTS AND DISCUSSION}

\section{Isolation and characterization of endogenously assembled mRNP complexes}

To develop a system allowing translation from highly purified genuine mRNP substrates, we began by assessing the composition of affinity-purified mRNPs assembled on tagged mRNAs expressed in human cells (Hogg and Collins 2007b; Hogg and Goff 2010). We used the PP7 coat protein and its cognate RNA hairpin binding site to isolate complexes associated with two different mRNAs containing GFP open reading frames: one with an unstructured $5^{\prime} \mathrm{UTR}$ that supports efficient translation (GFP-PP7) and one containing a strong cap-proximal RNA structure (HP-GFP-PP7; $\Delta \mathrm{G}=$ $-35 \mathrm{kcal} / \mathrm{mol}$ ) that prevents translation initiation (Fig. 1A, B; Supplemental Fig. 1A; Babendure et al. 2006). As a control, purifications were performed using extracts lacking tagged mRNAs (mock). Importantly, only minor background purification of proteins was observed in mock samples, indicating that the mRNPs were purified to near-homogeneity (Fig. 1B). Both SDS-PAGE and mass spectrometry revealed significant differences in composition between the two tagged mRNAs, consistent with their distinct translational states (Fig. 1B; Supplemental Table 1). Specifically, the translationally inac-
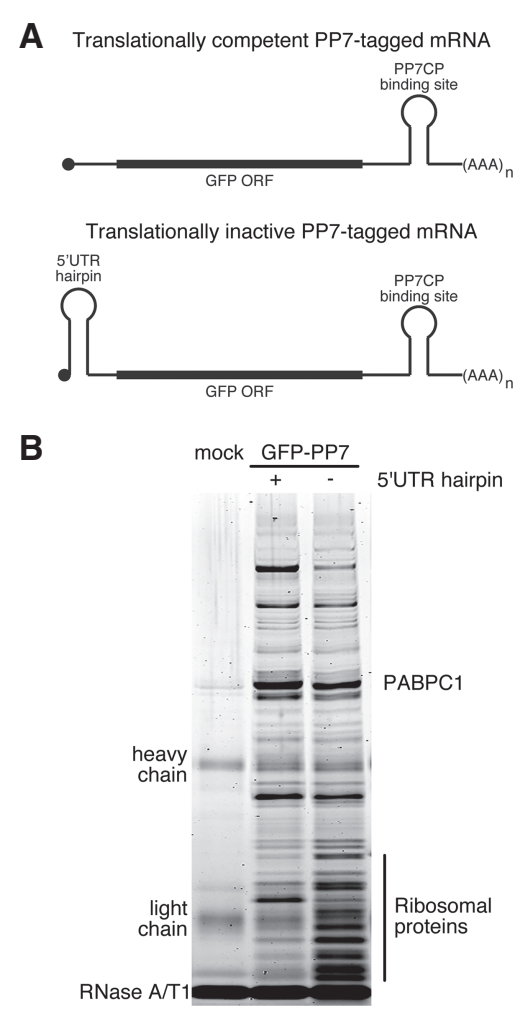

FIGURE 1. Isolation and characterization of endogenously assembled mRNPs. (A) Schematic of PP7-tagged mRNAs used as mRNP purification substrates. mRNAs with an unstructured $5^{\prime}$ UTR are efficiently translated (top), while mRNAs with a stable $5^{\prime}$ hairpin are translationally repressed (bottom). (B) SDS-PAGE gel stain of proteins copurified with PP7-tagged mRNAs with $(+)$ and without $(-)$ a cap-proximal structure that prevents translation initiation. Mock purifications from extracts lacking tagged mRNAs were performed as controls.

tive mRNAs were associated with fewer low-molecularweight ribosomal proteins, while recruiting several additional RNA-binding proteins. Mass spectrometry of the purified complexes identified more than 160 proteins represented by at least five peptide spectral counts in each sample. While the makeup of individual mRNPs within the purified population will be heterogeneous, this level of protein identification makes it possible to perform downstream functional assays on complexes whose typical composition has been determined with high confidence.

\section{Affinity-purified mRNPs are functional substrates for in vitro translation}

To allow for easy manipulation of translation reaction components, we elected to use established methods for in-house generation of translation-competent extracts from human tissue culture cells (Pisarev et al. 2010; Rakotondrafara and Hentze 2011). For the experiments described herein, actively dividing HEK-293 cells were harvested at subconfluency, and cytoplasmic extracts were prepared as previously described (Rakotondrafara and Hentze 2011). As substrates for in vitro 

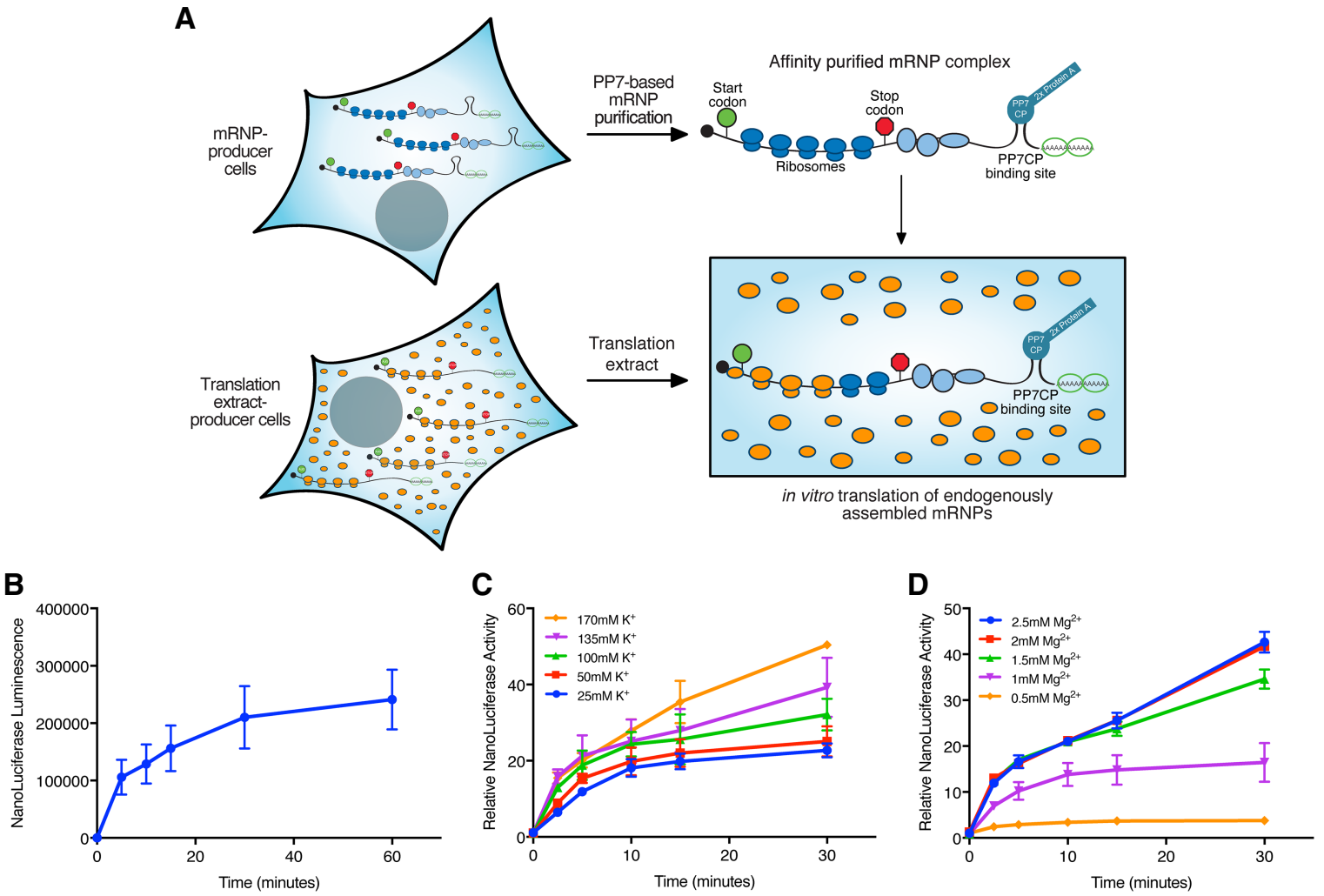

FIGURE 2. In vitro translation of endogenously assembled mRNPs under physiological buffer conditions. ( $A$ ) Scheme for in vitro translation of endogenously assembled mRNPs. Distinct cell populations were used to express and assemble PP7-tagged mRNAs into mRNPs (top) and produce translation-competent extracts (bottom). Following affinity purification, mRNPs immobilized on magnetic beads were introduced to extracts for cell-free translation. Translation can result both from copurified ribosomes (blue) and de novo initiation of ribosomes present in translation extracts (orange). (B) Affinity-purified NLuc mRNPs were incubated in translation extracts in the presence of $1.5 \mathrm{mM} \mathrm{Mg}^{2+}$ and $135 \mathrm{mM} \mathrm{K}$, and NLuc luminescence was monitored at the indicated time points. Results from seven independent experiments are shown; error bars indicate SD. (C) Affinity-purified NLuc mRNPs were translated under the indicated $\mathrm{K}^{+}$concentrations, with constant $1.5 \mathrm{mM} \mathrm{Mg}{ }^{2+}$. Results from two independent experiments are shown; error bars indicate SD. $(D)$ mRNP translation reactions as above performed with the indicated $\mathrm{Mg}^{2+}$ concentrations and $135 \mathrm{mM} \mathrm{K}{ }^{+}$. Results from two independent experiments are shown; error bars indicate SD.

translation of endogenously assembled mRNPs, we used PP7-tagged mRNAs encoding the small, bright, rapidly folding NLuc protein (Hall et al. 2012). We transfected HEK-293 cells with constructs expressing tagged NLuc mRNAs from the strong CMV promoter, prepared wholecell extracts, and isolated the tagged mRNPs using tandem protein A-tagged PP7 coat protein under similar conditions to those used for mass spectrometry. The mRNPs bound to magnetic beads were then transferred to translation extracts and incubated at $37^{\circ} \mathrm{C}$ for translation (Fig. 2A). Following the shift to $37^{\circ} \mathrm{C}$, we observed rapid increases in the NLuc signal, illustrating that this system can be used for quantitative assessment of translation occurring on endogenously assembled $\mathrm{mRNPs}$ with high temporal resolution (Fig. 2B).

\section{Maximal in vitro $\mathrm{mRNP}$ translation activity depends upon physiological $\mathrm{K}^{+}$and $\mathrm{Mg}^{2+}$ ionic concentrations}

The report describing the in vitro translation extracts used here recommended use of $50 \mathrm{mM}$ potassium acetate (KOAc) and $2.5 \mathrm{mM}$ magnesium acetate (MgOAc) as a starting point for optimization (Rakotondrafara and Hentze 2011). As these values deviate from commonly cited intracellular concentrations of $\sim 140 \mathrm{mM} \mathrm{K}^{+}$and $\sim 1 \mathrm{mM}$ free $\mathrm{Mg}^{2+}$, we next tested the mono- and divalent ion requirements for efficient translation of mRNP substrates (Hille 2001; Romani 2011). In these and subsequent experiments, we determined relative translation activity by calculating the fold increase in luminescence at the indicated time points. This approach corrects for differences in raw luminescence values caused by minor variability of mRNA expression levels among biological replicate transfections. In line with physiological conditions, increasing potassium concentration led to increased mRNP translation activity, up to the highest concentration tested of $170 \mathrm{mM}$ (Fig. 2C). Lowering $\mathrm{Mg}^{2+}$ from $2.5 \mathrm{mM}$ to $1.5 \mathrm{mM}$ had a minimal effect on translation activity, but concentrations below $1.5 \mathrm{mM}$ impaired NLuc production (Fig. 2D). Because physiologically relevant levels of potassium and magnesium ions ( $135 \mathrm{mM} \mathrm{K}^{+}$and $1.5 \mathrm{mM} \mathrm{Mg}^{2+}$ ) supported high levels of translation, we used these conditions for further experiments. 


\section{In vitro translation of purified mRNPs is much more efficient than standard IVT mRNA substrates}

To allow comparison to standard in vitro translation methods, we used T7 polymerase to transcribe the same NLuc mRNA sequence in vitro. The in vitro transcribed mRNAs (IVT mRNAs) were subsequently capped and polyadenylated to promote translation. In contrast to the behavior of the mRNP described above, translation of IVT mRNAs was strongly inhibited by physiological buffer conditions (Fig. $3 \mathrm{~A})$. For appropriate comparison of translation efficiencies
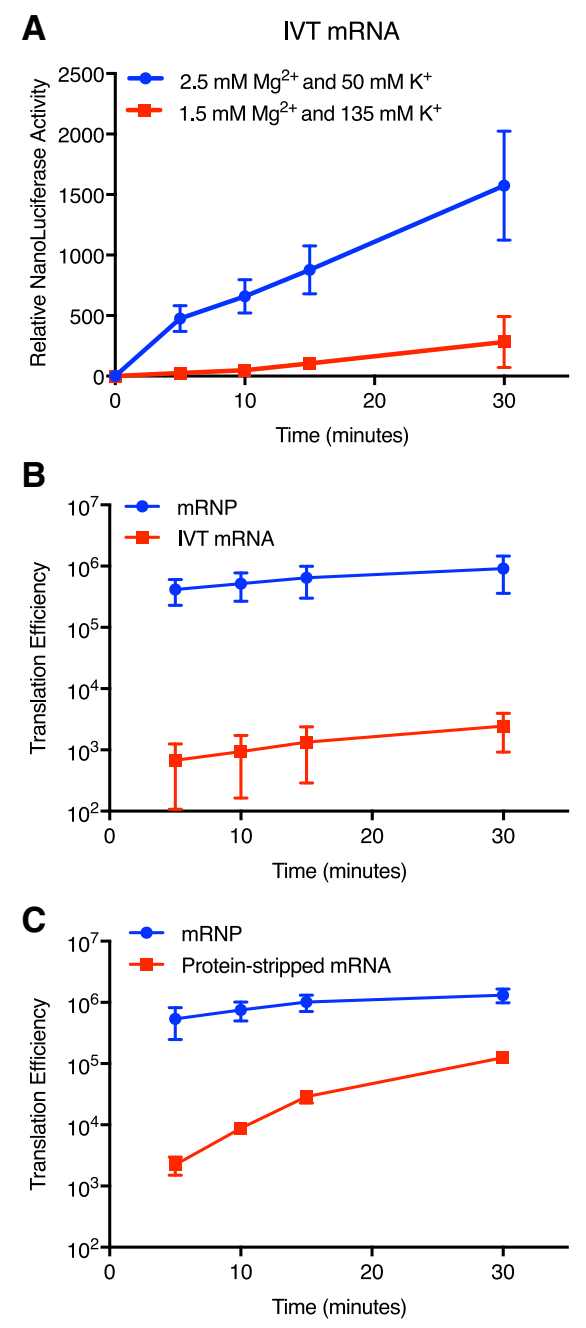

FIGURE 3. Translation of mRNPs is much more efficient than that of standard IVT mRNA substrates. (A) Quantification of IVT NLuc mRNA translation, with recommended $2.5 \mathrm{mM} \mathrm{Mg}^{2+}$ and $50 \mathrm{mM} \mathrm{K}^{+}$versus physiological $1.5 \mathrm{mM} \mathrm{Mg}^{2+}$ and $135 \mathrm{mM} \mathrm{K}^{+}$. Results from two independent experiments are shown; error bars indicate SD. $(B)$ NLuc activity produced by translation of mRNPs or in vitro transcribed mRNAs was normalized to mRNA content quantified by northern blotting to determine translation efficiency. Results from seven (mRNP) and four (IVT) independent experiments are shown; error bars indicate SD. (C) NLuc activity produced by translation of mRNPs or proteinstripped mRNAs was normalized to mRNA content quantified by northern blotting to determine translation efficiency. Results from four independent experiments are shown; error bars indicate SD. from IVT and mRNP substrates, we therefore used $50 \mathrm{mM}$ KOAc and $2.5 \mathrm{mM} \mathrm{MgOAc}$ for translation of IVT mRNAs. To determine the efficiency of translation from mRNP and IVT mRNA substrates, we quantified the amounts of mRNA present in mRNP purifications by northern blotting, using the IVT mRNA as a standard (Supplemental Fig. 1B). Division of NLuc values by the quantities of NLuc mRNA present in each reaction revealed that translation from the purified mRNPs was dramatically more efficient than from the IVT mRNAs, with a greater than 100-fold preference for translation from the mRNPs (Fig. 3B).

To test whether enhanced translation of the mRNP substrates could in part be due to proper capping, polyadenylation, or other covalent mRNA modifications, we phenol-extracted the mRNA from the affinity-purified mRNPs and subsequently introduced the protein-stripped mRNA to translation extracts. Similar to the IVT mRNA, affinity purified but protein-free mRNA was translated five- to 10 -fold more poorly under physiological ionic strength conditions than in buffer supplemented with $50 \mathrm{mM} \mathrm{KOAc}$ and $2.5 \mathrm{mM} \mathrm{MgOAc}$ (Supplemental Fig. 1C). Comparing translation of protein-stripped and IVT mRNAs translated under low-ionic strength conditions to translation of purified mRNPs under physiological ionic conditions, the endogenously expressed mRNA was translated with greater efficiency than the capped and polyadenylated IVT mRNA, but was still much less efficiently translated than the intact mRNP substrates, particularly at early time points (Fig. 3B,C; Supplemental Fig. 1D). These data indicate that endogenous mRNA capping, polyadenylation, or other covalent modifications contribute to enhanced translation efficiency of purified mRNPs, but that optimal translation of mRNPs under physiological conditions requires copurified protein components.

\section{Translation activity of purified mRNPs is attributable to both elongation from copurified ribosomes and de novo initiation}

Our mass spectrometry experiments indicated that $80 \mathrm{~S}$ ribosomes could be copurified with translation-competent tagged mRNPs, suggesting that the mRNPs were bound to ribosomes in the act of translation (Figs. 1B, 4A; Supplemental Table 1). Further, we noticed that the initial rates of NLuc production from the mRNP and IVT mRNA substrates were different, with the mRNP immediately producing active NLuc protein and the IVT mRNA expressing active NLuc after a lag period of $2.5 \mathrm{~min}$ (Fig. 4B). These findings suggested that translation of the mRNPs could be divided into two phases: elongation from copurified ribosomes and de novo initiation of ribosomes present in the translation extracts.

We next devised strategies to disrupt different steps in mRNP translation to test this hypothesis and develop approaches capable of studying translation performed by these distinct pools of ribosomes (Fig. 4A). To abolish the contribution of run-off translation from mRNP-bound ribosomes, 
A
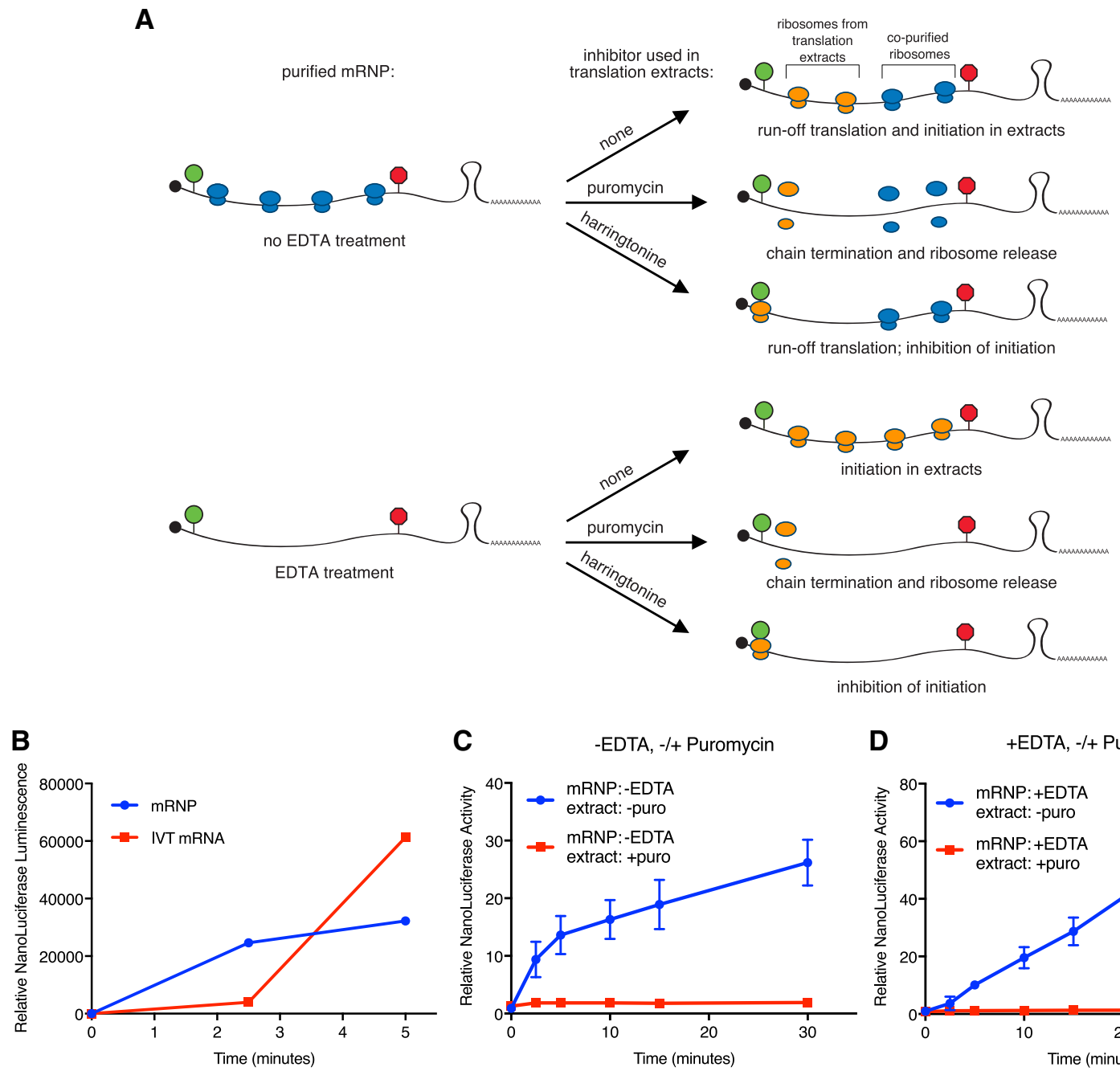

C

-EDTA, -/+ Puromycin

D

+EDTA, -/+ Puromycin
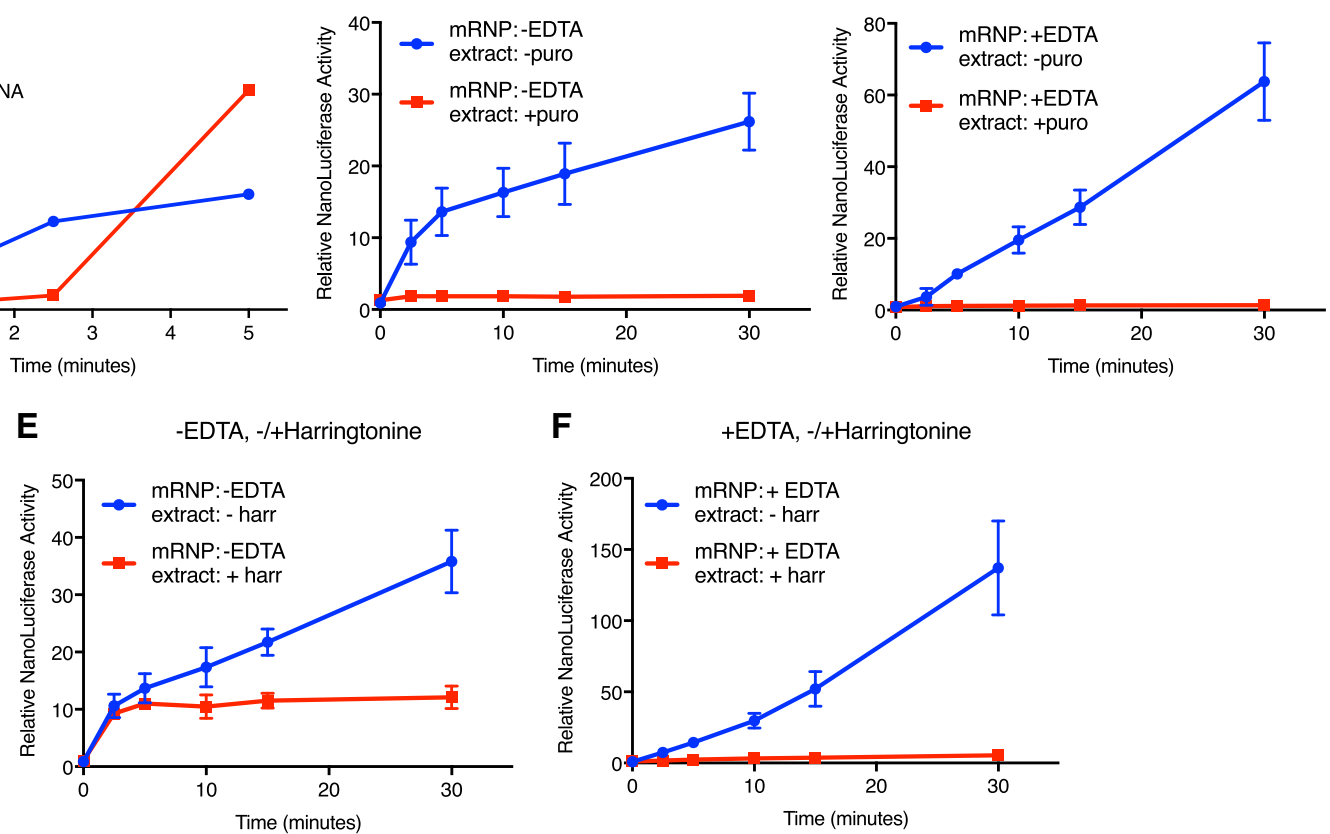

FIGURE 4. mRNP substrates for in vitro translation allow independent analysis of run-off translation and de novo initiation. (A) Scheme for mRNP purification and in vitro translation to separate run-off translation from de novo initiation. mRNP complexes were affinity purified in the absence or presence of EDTA and then introduced to cell-free extracts with no translation inhibitor added, puromycin, or harringtonine. The effects of these treatment conditions on run-off translation versus de novo initiation are shown on the right. Ribosomes copurified with mRNPs are shown in blue, while ribosomes provided by cytoplasmic translation extracts are orange. (B) Representative data from Figure 4E and Supplemental Figure 2C, expanded to show initial rates of mRNP translation during the first $5 \mathrm{~min}$. (C) Quantification of NLuc mRNP translation, with mRNPs purified in the absence of EDTA treatment and translation extracts supplemented with puromycin $(+)$ or $\mathrm{ddH}_{2} \mathrm{O}(-)$. Results from two independent experiments are shown; error bars indicate SD. (D) Quantification of NLuc mRNP translation, with mRNPs purified in the presence of EDTA and translation extracts supplemented with puromycin $(+)$ or $\mathrm{ddH}_{2} \mathrm{O}(-)$. Results from two independent experiments are shown; error bars indicate SD. (E) Quantification of NLuc mRNP translation, with mRNPs purified in the absence of EDTA treatment and translation extracts supplemented with harringtonine $(+)$ or DMSO (-). Results from two independent experiments are shown; error bars indicate SD. $(F)$ Quantification of NLuc mRNP translation, with mRNPs purified in the presence of EDTA and translation extracts supplemented with harringtonine $(+)$ or DMSO $(-)$. Results from two independent experiments are shown; error bars indicate SD. 
we treated the purified mRNPs with EDTA prior to their incubation with translation extracts. Either alone or with EDTA pretreatment of purified mRNPs, we also treated translation extracts with puromycin, an aminoacyl tRNA analog that binds the ribosome A-site and causes premature chain termination and ribosome release to inhibit all translation elongation (Cannon 1968; Blobel and Sabatini 1971). Similarly, we inhibited initiation in translation extracts with harringtonine, which traps initiated $80 \mathrm{~S}$ complexes at the start codon by blocking recruitment of subsequent aminoacyl tRNAs and interfering with peptidyl-transferase activity (Fresno et al. 1977).

As expected, puromycin treatment completely prevented translation from the mRNPs, whereas EDTA pretreatment disrupted the initial phase of mRNP translation but allowed robust activity over time (Fig. 4C,D; Supplemental Fig. 2A,B). Translation of IVT mRNAs was completely inhibited by both puromycin and harringtonine, as protein synthesis is limited to de novo initiation on these mRNAs (Supplemental Fig. 2C, D). Importantly, harringtonine treatment of extracts used to translate mRNPs purified with intact ribosomes (i.e., -EDTA) allowed an initial burst of translation activity followed by a plateau, consistent with the hypothesis that the initial phase of mRNP translation is due to elongation by copurified ribosomes. In contrast, harringtonine prevented any translation of mRNPs that had been pretreated with EDTA (Fig. 4E,F). Together, these results suggest that the initial phase of translation from mRNPs is driven by run-off translation from copurified ribosomes, after which initiation by extract-provided ribosomes predominates. Moreover, these data indicate that, while maximal translation of purified mRNPs involves both run-off and de novo translation, high translation efficiency of mRNP substrates is also achieved when all translation is initiated by extract-derived ribosomes (Supplemental Fig. 2A,B).

The NLuc mRNAs used in the preceding experiments are minimal transcripts containing a short, vector-derived $3^{\prime}$ UTR. To test whether the use of mRNPs as substrates also promotes translation of mRNAs with more complex features, we constructed translation reporters in which the NLuc ORF was introduced to a widely used $\beta$ globin minigene containing two efficiently spliced introns and either the $111 \mathrm{nt}$ $\beta$-globin $3^{\prime}$ UTR or the $\sim 1.3 \mathrm{~kb} \mathrm{3}$ UTR of the human nonsense-mediated decay factor SMG5 (Lykke-Andersen et al. 2000; Singh et al. 2008; Hogg and Goff 2010; Ge et al. 2016). As with the minimal NLuc mRNAs, the spliced $\beta$ globin mRNAs were much more efficiently translated from either untreated or EDTA-pretreated mRNPs than from IVT mRNAs (Fig. 5A,B; Supplemental Fig. 2E,F). Interestingly, the magnitude of translation enhancement observed with mRNP translation was similar across all of the mRNAs tested, even though the $\beta$ globin-derived mRNAs were less efficiently translated than the minimal NLuc mRNAs in each condition (cf. Fig. 5A,B to Fig. 3B and Supplemental Fig. 2C). Together, these findings show that mRNAs with distinct sequences and features are preferentially translated as affinity-purified mRNPs and suggest that this method will serve as a flexible tool for studies of translation.

\section{Conclusions}

Together, our data show that affinity-purified mRNP complexes assembled in vivo can be used as highly efficient substrates for in vitro translation under physiological conditions.
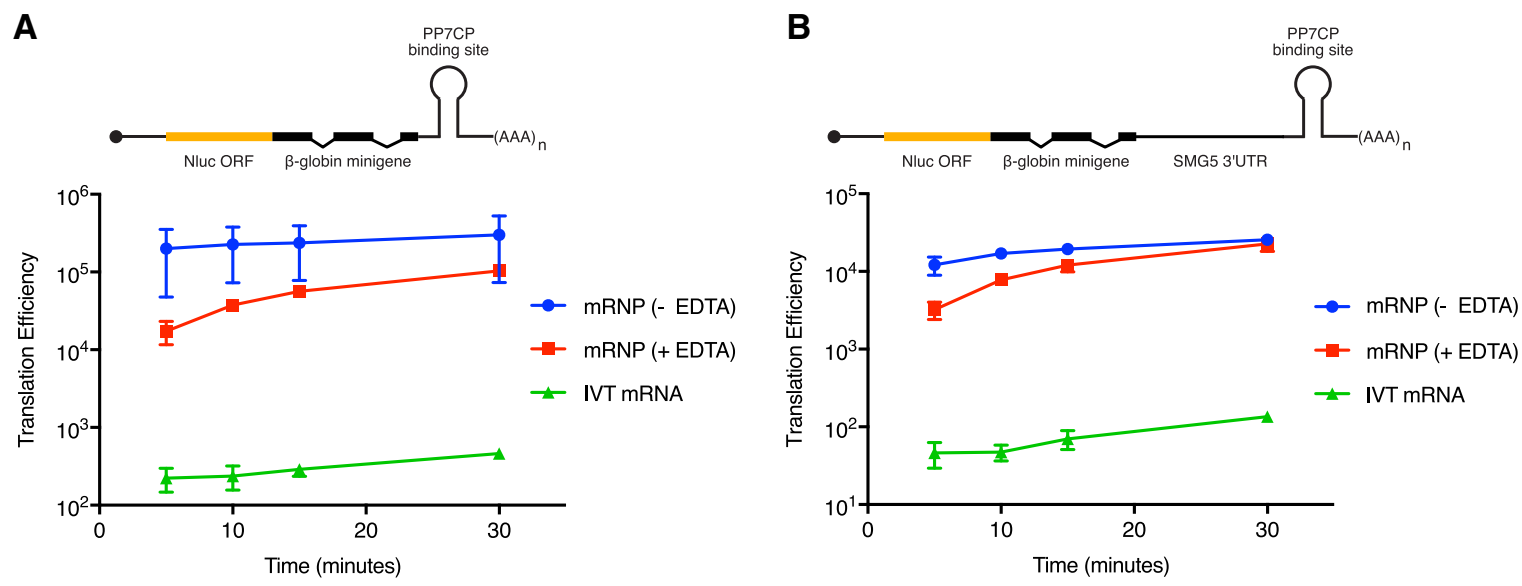

FIGURE 5. mRNP substrates with more complex features also promote translation in vitro. (A) Schematic of the PP7-tagged NLuc $\beta$ wt $\beta$ TP mRNA used as a mRNP purification substrate (top). NLuc activity produced by translation of the NLuc $\beta \mathrm{wt} \beta \mathrm{TP}$ mRNPs in the presence $(+)$ or absence $(-)$ of EDTA or the corresponding in vitro transcribed mRNAs was normalized to mRNA content quantified by northern blotting to determine translation efficiency (bottom). Results from two (-EDTA) and five (+EDTA and IVT) independent experiments are shown; error bars indicate SD. (B) Schematic of the PP7-tagged NLuc $\beta$ wtSMG5TP mRNA used as a mRNP purification substrate (top). NLuc activity produced by translation of the NLuc $\beta$ wtSMG5TP mRNPs in the presence $(+)$ or absence $(-)$ of EDTA or the corresponding in vitro transcribed mRNAs was normalized to mRNA content quantified by northern blotting to determine translation efficiency (bottom). Results from two (-EDTA) and four (+EDTA and IVT) independent experiments are shown; error bars indicate SD. 
A combination of this approach with mass spectrometry allows sensitive determination of the protein composition of the purified mRNPs, which can in turn be readily controlled by depletion or overexpression of factors in the cells used to express the tagged mRNAs. We expect that this flexibility will enable mechanistic studies of the impact of specific mRNAbinding proteins and RNA modifications in the regulation of translation and mRNA stability. Further, this system can be manipulated with standard small molecule inhibitors of translation to isolate distinct phases of translation and to study contributions of copurified and extract-derived ribosomes. These findings suggest that this approach may also be useful for studies of specialized ribosomes and tissuespecific ribosome-associated factors (Shi and Barna 2015).

\section{MATERIALS AND METHODS}

\section{Plasmids}

Plasmids for expression of PP7-tagged GFP mRNAs and the PP7 coat protein were previously described (Hogg and Goff 2010). The NLuc ORF (Promega) was introduced into the HindIII-XbaI sites of pcDNAGFPTP, replacing the GFP ORF. For $\beta$ globin minigenederived plasmids, the NLuc ORF was introduced into the HindIII site of $\mathrm{pc} \beta \mathrm{wt} \beta$ (Lykke-Andersen et al. 2000) or pc $\beta w t S M G 5 T P$ (Ge et al. 2016) by the sequence and ligation-independent cloning method (Li and Elledge 2007), such that the $\beta$ globin $5^{\prime}$ UTR was deleted and the NLuc ORF was fused in frame to the $\beta$ globin ORF. To allow mRNP purification, the PP7 hairpin was inserted into the XbaI site of the resulting pcNLuc $\beta w t \beta$ plasmid, downstream from the wild-type $\beta$ globin 3'UTR.

\section{Cell culture and transfections}

For in vitro translation reactions, HEK-293 Tet-off cells (632107; Clontech) were cultured in DMEM (11965-092; Gibco) supplemented with 10\% FBS (A31606-02; Gibco) and 1\% penicillin/ streptomycin with L-Glutamine (10378-016; Gibco). Transfection of cells with pcNLucTP, pcNLuc $\beta w t \beta T P$, or pcNLuc $\beta w t S M G 5 T P$ was performed by calcium phosphate as previously described (Hogg and Collins 2007a,b). Expression of the PP7-tagged GFP mRNAs was validated by cotransfecting HEK-293 Tet-off cells with pLVX-mCherry plasmid (Clontech) using Lipofectamine 3000 Reagent (Invitrogen). Cells were harvested 24-h post-transfection in $1 \times$ Passive Lysis Buffer (Promega) and the levels of GFP and mCherry fluorescence detected using an Infinite F200 Pro microplate reader and associated i-control 1.9 software (Tecan).

\section{mRNP purification and preparation of IVT RNA}

PP7-based mRNP purification was performed as previously described (Hogg and Goff 2010). For SDS-PAGE, complexes were eluted with RNase A/T1 Cocktail (AM2286; Ambion), diluted 1:50 in HLB150, as previously described (Hogg and Goff 2010). For mass spectrometry, samples were eluted in HLB supplemented with $1 \mathrm{M} \mathrm{NaCl}$. For isolation of protein-stripped mRNAs, PP7based mRNP purifications were performed and mRNA phenol- extracted using TRIzol (15596026; Invitrogen). Capped and polyadenylated in vitro transcribed NLuc RNA was prepared using ApaIlinearized NLuc template DNA and the mMessage mMachine T7 Transcription (AM1344; Ambion) and Poly(A) Tailing (AM1350; Ambion) kits according to manufacturer instructions. NLuc $\beta w t \beta T P$ and NLuc $\beta$ wtSMG5TP IVT mRNAs were prepared using PCRamplified cDNA as a template for the in vitro transcription reactions (PCR primers: 5' Forward: TAA TAC GAC TCA CTA TAG GGA GAC; 3' Reverse: AGG AAA GGA CAG TGG GAG TG).

\section{Generation of translation-competent cytoplasmic extract}

HEK-293 Tet-off translation-competent cytoplasmic extract was generated as previously described (Rakotondrafara and Hentze 2011) with the following modification: A 27G 1/1/4-in needle (305136; BD PrecisionGlide) was used to homogenize the cells.

\section{In vitro translation}

In vitro translation assays were conducted by adapting the protocol described by Rakotondrafara and Hentze (see Supplemental Protocol for details; Rakotondrafara and Hentze 2011). A total reaction volume of $50 \mu \mathrm{L}$ per sample was prepared on ice as follows: $16 \mathrm{mM}$ HEPES-KOH, pH 7.6, $6.55 \mathrm{mg} / \mathrm{mL}$ creatine phosphate (10621722001; Roche), $0.1 \mathrm{mg} / \mathrm{mL}$ creatine kinase (10127566001; Roche), 0.8 mM ATP (P0756S; New England BioLabs), $0.1 \mathrm{mM}$ spermidine, $0.1 \mathrm{mM}$ complete amino acid mix (L4461; Promega), $135 \mathrm{mM}$ potassium acetate (for purified mRNPs) or $50 \mathrm{mM}$ potassium acetate (for IVT mRNA and protein-stripped PP7-purified mRNA), $1.5 \mathrm{mM}$ magnesium acetate (for purified mRNP) or $2.5 \mathrm{mM}$ magnesium acetate (for IVT mRNA and protein-stripped PP7-purified mRNA), and $20 \mu \mathrm{L}$ of equilibrated $(10 \mathrm{mg} / \mathrm{mL})$ translation-competent cytoplasmic extract.

Following the final wash in HLB150 $+0.1 \%$ NP-40, purified mRNPs were washed once in translation wash buffer $(10 \mathrm{mM}$ HEPES-KOH, pH 7.6, $135 \mathrm{mM}$ potassium acetate, $1.5 \mathrm{mM}$ magnesium acetate) then immediately resuspended in the $50 \mu \mathrm{L}$ of prepared translation reaction. A $6 \mu \mathrm{L}$ aliquot was reserved in a prechilled microcentrifuge tube on ice as time point 0 and the remaining reaction directly transferred to a $37^{\circ} \mathrm{C}$ ThermoMixer (5382000015; Eppendorf) with ThermoTop lid (5308000003; Eppendorf) set to shake at $1500 \mathrm{rpm}$ for $15 \mathrm{sec}$ every $2 \mathrm{~min}$. At indicated time points, $6 \mu \mathrm{L}$ aliquots were taken and placed into prechilled microcentrifuge tubes on ice as conducted at time point 0 . For IVT mRNA reactions, 0.5 picomoles of the corresponding IVT mRNA was added to the $50 \mu \mathrm{L}$ prepared translation reaction, and in vitro translation was conducted as with the purified mRNPs. For experiments with translation inhibitors, $2 \mu \mathrm{L}$ of $1 \mathrm{mg} / \mathrm{mL}$ harringtonine (H0169; LKT Laboratories) or $1 \mu \mathrm{L}$ of $1 \mathrm{mg} / \mathrm{mL}$ puromycin (P9620; Sigma-Aldrich) were added to each $50 \mu \mathrm{L}$ translation reaction. To examine the effect of run-off translation, $25 \mathrm{mM}$ (final) EDTA was included during the PP7-based mRNP purification.

To determine the amount of NLuc produced at each time point, $2 \mu \mathrm{L}$ of the reserved reaction was diluted in $50 \mu \mathrm{L}$ of $\mathrm{ddH}_{2} \mathrm{O}$ and then combined with prepared NanoGlo Luciferase Assay Reagent (N1130; Promega) in a 96-well white-bottom plate (655904; Greiner Bio-one) according to manufacturer instructions. NLuc luminescence was detected using an Infinite F200 Pro microplate 
reader and associated i-control 1.9 software (Tecan). NLuc luminescence was determined by subtracting the background at time 0 from each individual time point within a sample (Figs. 2B, 4B). Translation efficiency was calculated by dividing the backgroundsubtracted NLuc luminescence by the amount of recovered RNA within an experiment (Figs. 3B,C, 5A,B; Supplemental Fig. 2A). Relative NLuc activity values indicate fold change in luminescence and were determined by dividing the NLuc luminescence at each time point by the average at time 0 across samples within a trial to adjust for minor fluctuations in mRNA expression and recovery (Figs. 2C,D, 3A, 4C-F; Supplemental Fig. 2C,D).

\section{Northern blotting}

One-tenth of the washed mRNP bead complexes was reserved prior to addition to the in vitro translation reaction and RNA isolated with TRIzol. Samples were resolved on formaldehyde/agarose gels and a

${ }^{32} \mathrm{P}$-labeled in vitro transcribed probe against NLuc was used for detection of mRNAs. Northern blots were imaged on a Typhoon scanner (GE Healthcare) and quantification was performed using ImageStudio software (LI-COR).

\section{Mass spectrometry}

Purified mRNPs were precipitated in cold acetone (Thermo Scientific), subjected to trypsin digestion, and analyzed on a LTQ Orbitrap Velos mass spectrometer (Thermo Electron), as previously described (Ge et al. 2016). MASCOT software (Matrix Science) was used to determine peptide and protein identities, and Scaffold (Proteome Software) was used for further analysis. Proteins identified by five or more spectral counts were considered putative mRNP components.

\section{SUPPLEMENTAL MATERIAL}

Supplemental material is available for this article.

\section{ACKNOWLEDGMENTS}

We thank Stacey Baker for assistance in establishing the mRNP translation method and members of the Hogg and Ferré-D'Amaré laboratories and Nicholas Guydosh for helpful discussions and critical reading of the manuscript. Mass spectrometry was performed by Stephen Swatkoski and Marjan Gucek in the NHLBI Proteomics Core. This work was supported by the Intramural Research Program, National Institutes of Health, National Heart, Lung, and Blood Institute.

Received January 17, 2018; accepted April 25, 2018.

\section{REFERENCES}

Babendure JR, Babendure JL, Ding J-H, Tsien RY. 2006. Control of mammalian translation by mRNA structure near caps. RNA 12: 851-861.

Blobel G, Sabatini D. 1971. Dissociation of mammalian polyribosomes into subunits by puromycin. Proc Natl Acad Sci 68: 390-394.
Cannon M. 1968. The puromycin reaction and its inhibition by chloramphenicol. Eur J Biochem 7: 137-145.

Chazal P-E, Daguenet E, Wendling C, Ulryck N, Tomasetto C, Sargueil B, Le Hir H. 2013. EJC core component MLN51 interacts with eIF3 and activates translation. Proc Natl Acad Sci 110: 5903-5908.

Fresno M, Jiménez A, Vázquez D. 1977. Inhibition of translation in eukaryotic systems by harringtonine. Eur J Biochem 72: 323-330.

Ge Z, Quek BL, Beemon KL, Hogg JR. 2016. Polypyrimidine tract binding protein 1 protects mRNAs from recognition by the nonsensemediated mRNA decay pathway. Elife 5: e11155.

Gehring NH, Wahle E, Fischer U. 2017. Deciphering the mRNP code: RNA-bound determinants of post-transcriptional gene regulation. Trends Biochem Sci 42: 369-382.

Hall MP, Unch J, Binkowski BF, Valley MP, Butler BL, Wood MG, Otto P, Zimmerman K, Vidugiris G, Machleidt T, et al. 2012. Engineered luciferase reporter from a deep sea shrimp utilizing a novel imidazopyrazinone substrate. ACS Chem Biol 7: 1848-1857.

Hille B. 2001. Ion channels of excitable membranes, 3rd ed. Sinauer Associates, MA.

Hogg JR, Collins K. 2007a. Human Y5 RNA specializes a Ro ribonucleoprotein for $5 \mathrm{~S}$ ribosomal RNA quality control. Genes Dev 21: 3067-3072.

Hogg JR, Collins K. 2007b. RNA-based affinity purification reveals 7SK RNPs with distinct composition and regulation. RNA 13: 868-880.

Hogg JR, Goff SP. 2010. Upf1 senses 3'UTR length to potentiate mRNA decay. Cell 143: 379-389.

Katzen F, Chang G, Kudlicki W. 2005. The past, present and future of cell-free protein synthesis. Trends Biotechnol 23: 150-156.

Li MZ, Elledge SJ. 2007. Harnessing homologous recombination in vitro to generate recombinant DNA via SLIC. Nat Methods 4: 251-256.

Lykke-Andersen J, Shu MD, Steitz JA. 2000. Human Upf proteins target an mRNA for nonsense-mediated decay when bound downstream of a termination codon. Cell 103: 1121-1131.

Mitchell SF, Parker R. 2014. Principles and properties of eukaryotic mRNPs. Mol Cell 54: 547-558.

Moore MJ. 2005. From birth to death: the complex lives of eukaryotic mRNAs. Science 309: 1514-1518.

Panchapakesan SSS, Ferguson ML, Hayden EJ, Chen X, Hoskins AA, Unrau PJ. 2017. Ribonucleoprotein purification and characterization using RNA Mango. RNA 23: 1592-1599.

Panthu B, Mure F, Gruffat H, Ohlmann T. 2015. In vitro translation of mRNAs that are in their native ribonucleoprotein complexes. Biochem J 472: 111-119.

Pisarev AV, Skabkin MA, Pisareva VP, Skabkina OV, Rakotondrafara AM, Hentze MW, Hellen CUT, Pestova TV. 2010. The role of ABCE1 in eukaryotic posttermination ribosomal recycling. Mol Cell 37: 196-210.

Rakotondrafara AM, Hentze MW. 2011. An efficient factor-depleted mammalian in vitro translation system. Nat Protoc 6: 563-571.

Romani AMP. 2011. Cellular magnesium homeostasis. Arch Biochem Biophys 512: 1-23.

Shi Z, Barna M. 2015. Translating the genome in time and space: specialized ribosomes, RNA regulons, and RNA-binding proteins. Annu Rev Cell Dev Biol 31: 31-54.

Shoemaker CJ, Green R. 2012. Translation drives mRNA quality control. Nat Struct Mol Biol 19: 594-601.

Singh G, Rebbapragada I, Lykke-Andersen J. 2008. A competition between stimulators and antagonists of Upf complex recruitment governs human nonsense-mediated mRNA decay. PLoS Biol 6: e111.

Slobodin B, Gerst JE. 2010. A novel mRNA affinity purification technique for the identification of interacting proteins and transcripts in ribonucleoprotein complexes. RNA 16: 2277-2290.

Yoon JH, Gorospe M. 2016. Identification of mRNA-interacting factors by MS2-TRAP (MS2-tagged RNA affinity purification). Methods Mol Biol 1421: 15-22. 

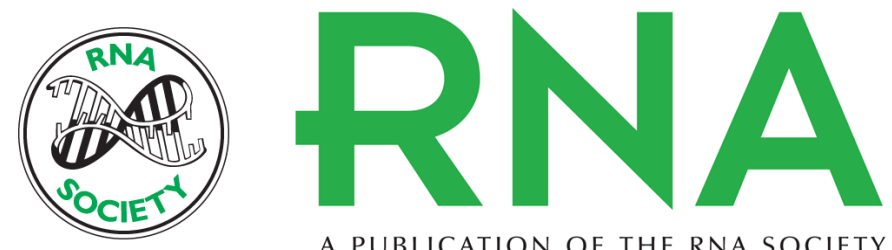

A PUBLICATION OF THE RNA SOCIETY

\title{
Highly efficient in vitro translation of authentic affinity-purified messenger ribonucleoprotein complexes
}

\author{
Sarah E. Fritz, Nazmul Haque and J. Robert Hogg
}

RNA 2018 24: 982-989 originally published online May 3, 2018

Access the most recent version at doi:10.1261/rna.065730.118

\section{Supplemental http://rnajournal.cshlp.org/content/suppl/2018/05/03/rna.065730.118.DC1 \\ Material}

References This article cites 26 articles, 9 of which can be accessed free at: http://rnajournal.cshlp.org/content/24/7/982.full.html\#ref-list-1

License This is a work of the US Government.

Email Alerting Receive free email alerts when new articles cite this article - sign up in the box at the Service top right corner of the article or click here.

\section{|||||||| Providing Precise Solutions for your research.}

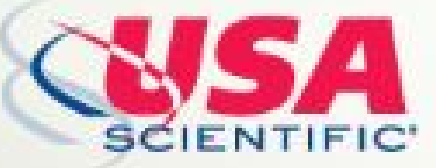

To subscribe to $R N A$ go to:

http://rnajournal.cshlp.org/subscriptions

Published by Cold Spring Harbor Laboratory Press for the RNA Society 